\title{
Linx
}

Revue des linguistes de l'université Paris X Nanterre

\section{Un thème, deux thèmes, un préambule ? Intonation, geste et morphosyntaxe dans le dialogue oral en français}

\section{Mary-Annick Morel}

\section{OpenEdition}

\section{Journals}

Édition électronique

URL : http://journals.openedition.org/linx/466

DOI : $10.4000 / \operatorname{linx} .466$

ISSN : 2118-9692

Éditeur

Presses universitaires de Paris Nanterre

\section{Édition imprimée}

Date de publication : 1 décembre 2006

Pagination : 133-152

ISSN : 0246-8743

\section{Référence électronique}

Mary-Annick Morel, « Un thème, deux thèmes, un préambule ? Intonation, geste et morphosyntaxe dans le dialogue oral en français ", Linx [En ligne], 55 | 2006, mis en ligne le 23 février 2011, consulté le 19 avril 2019. URL : http://journals.openedition.org/linx/466 ; DOI : 10.4000/linx.466 


\title{
Un thème, deux thèmes, un préambule? Intonation, geste et morphosyntaxe dans le dialogue oral en français
}

\author{
Mary-Annick Morel \\ Université Paris 3 - Sorbonne Nouvelle \\ EA 1483 Recherche sur le français contemporain \\ marym@ext.jussieu.fr
}

Les hypothèses théoriques développées dans l'EA 1483 de Paris 3 pour l'analyse des corpus d'oral spontané sont le fruit d'une recherche pluridisciplinaire associant analyse morphosyntaxique et lexicale, interprétation des tracés mélodiques, examen minutieux de la mimique-gestuelle, et théorie de la coénonciation. La recherche est fondée sur des discours oraux relativement longs recueillis dans des situations de parole différentes : discussions à bâtons rompus, interviews, entretiens radiophoniques, dialogues argumentatifs, récits, interactions de service, consultations d'expert, exposés didactiques, etc. ${ }^{1}$ La spécificité de la démarche réside dans la prise en compte conjointe des indices intonatifs (variations de F0, de l'intensité et de la durée, position et longueur des pauses silencieuses) et de la morphosyntaxe et, chaque fois que le corpus le permet, des indices mimico-gestuels (mouvement de la tête et du regard, gestes des mains).

\footnotetext{
${ }^{1}$ Les enregistrements (plus de 300 enregistrements différents de 30 minutes à une heure) ont été en majeure partie réalisés par les étudiants du séminaire sur l'oral de l'EA 1483 «Recherche sur le français contemporain» de Paris 3. Qu'ils soient ici remerciés pour leur précieuse collaboration et pour leur enthousiasme constructif.
} 
Mary-Annick Morel

\section{Méthodologie de l’analyse}

\subsection{Analyse de l'intonation}

Pour transformer en données observables les données brutes fournies par les tracés mélodiques, nous commençons par identifier le registre intonatif de chaque locuteur sur une séquence d'environ trois minutes de parole dans laquelle se trouve inséré l'extrait étudié, ce qui ne préjuge en rien de la plage mélodique de ces mêmes locuteurs à un autre moment de l'échange ou dans d'autres situations de discours. A l'intérieur de cette séquence, nous déterminons le point le plus haut et le point le plus bas, à partir du milieu de cette plage de variations mélodiques nous identifions la plage haute et la plage basse. Cette procédure permet de faire ressortir un certain nombre de constantes d'un tracé à l'autre et d'un corpus à l'autre :

- le niveau le plus bas correspond à la finale absolue d'une intervention, et souvent à celle d'un paragraphe oral,

- le niveau le plus haut marque la hauteur finale d'un segment ayant valeur de préambule ou encore celle d'une interrogation ou d'un constituant focalisé.

Pour l'interprétation globale de la valeur coénonciative des constituants, les variations de $\mathrm{F} 0$ en plage haute traduisent un mouvement iconique d'appel à l'autre et une prise en compte de la coénonciation, alors que les variations en plage basse marquent de façon iconique un repli sur soi, une sortie de la coénonciation, une attitude égocentrée.

$$
\begin{aligned}
& \text { Haut }=\text { mise en jeu de la coénonciation (convergence/discordance) } \\
& \text { = consensualité acquise plage haute } \\
& =\text { repli sur soi }
\end{aligned}
$$

Lecture des tracés mélodiques obtenus avec ANAPROZ (logiciel PC conçu par François Colombo, ingénieur en automatique, spécialisé dans le dialogue Homme-Machine $)^{2}$

La courbe du haut représente les variations d'intensité ${ }^{3}$. La courbe du milieu représente les variations de la mélodie. La première ligne donne ici F0 à 100 Hertz et la ligne la plus haute est étalonnée à 400 Hertz; mais si les variations mélodiques de la locutrice montent effectivement à $400 \mathrm{~Hz}$, en revanche elles ne descendent que peu en dessous de $200 \mathrm{~Hz}$. La plage basse de la locutrice situe donc entre 180 et $290 \mathrm{~Hz}$ et la plage haute entre 290 et $400 \mathrm{~Hz}$. La ligne au dessous de la bande de transcription donne la durée. Les chiffres indiquent les secondes, et chaque trait de subdivision correspond à dix centisecondes (une déciseconde). Ces chiffres servent à repérer le segment de discours pris en considération dans les analyses.

\footnotetext{
${ }^{2}$ Les étudiants de Paris 3 utilisent actuellement les logiciels Praat, Winpitch ou Speechanalyser.

${ }^{3}$ Codage des indices intonatifs : $\mathrm{F} 0=$ fondamental de la voix (mélodie) $/ \mathrm{D}=$ durée en centisecondes $(\mathrm{cs}) / \mathrm{I}=$ intensité $/\{\mathrm{xx}\}=$ durée de la pause silence en cs. Les intervalles chiffrés donnés dans les exemples — $\{8-9\}$ ou $\{10-11\}$ — correspondent à la durée en seconde marquée sur la ligne du bas dans les tracés mélodiques.
} 
(1) Corpus CRS : Au cours d'un dialogue portant sur la sécurité dans le métro, une étudiante Jane rapporte à une camarade qu'elle connait bien, Tatiana, les mésaventures d'une jeune femme qui n'avait pas son titre de transport.

Tracé 1 : (8-9) donc c'est une nana (8-10) elle prend l'métro (10-11) et elle a pas son billet ${ }^{4}$

Tracé 1 : début du récit sur les CRS dans le métro

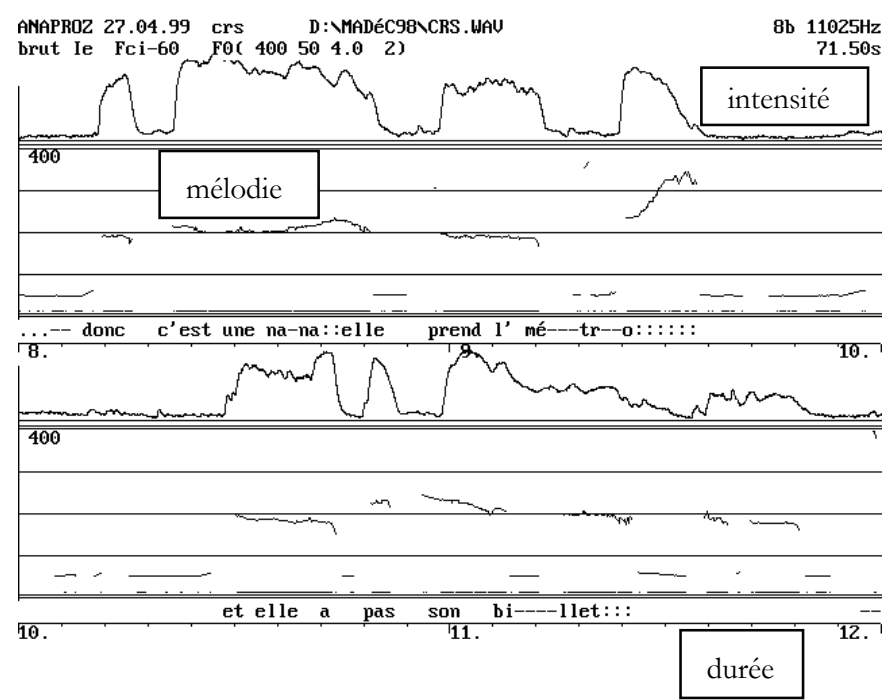

Le point de départ de la recherche repose sur deux postulats de base.

(1) Le français obéit à un principe général de décondensation. Ce principe explique entre autres la juxtaposition (dans le préambule) d'un certain nombre de constituants dissociés de la construction syntaxique réalisée par le verbe du rhème.

(2) Il n'y a pas redondance mais complémentarité entre les indices intonatifs et les marqueurs morphosyntaxiques et lexicaux.

Les marques morphosyntaxiques et les marques intonatives sont réparties de part et d'autre du lexème constructeur des segments successifs du discours oral. Les marqueurs morphosyntaxiques (déterminant pour les noms, préposition pour les groupes prépositionnels, pronom sujet pour les verbes) se situent à l'initiale, explicitant ainsi l'identité du syntagme, cf. Tracé 1 donc c'est une nana elle prend l'métro $\{60\}$ et elle a pas son billet. Les variations intonatives affectent au contraire la syllabe finale des

${ }^{4}$ Dans tous les exemples, le préambule est donné en italiques et le rhème en caractères droits (voir ci-après \$3.1.)

${ }^{5}$ Conventions de transcription : $\{\mathrm{xx}\}$ durée de la pause en centisecondes / $\mathrm{x}:$ ou x:: allongement de la durée de la voyelle / (h) reprise de respiration audible / e ou e:: 'euh' d'hésitation $/{ }^{\circ} \mathrm{xxx}^{\circ}$ incise $=$ décrochement intonatif en plage basse $/ \llbracket$ ou soulignement $=$ recouvrement de paroles $/ \int \mathrm{m} \$ ou $\ \mathrm{~mm} \$ manifestation sonore de l'écouteur $/$ syllabe en exposant $=$ montée mélodique $/$ syllabe en indice $=$ chute de la mélodie 
Mary-Annick Morel

constituants discursifs, auxquels elles assignent une fonction spécifique dans le marquage des relations intersubjectives et dans la construction du discours.

\subsection{Analyse de la mimique-gestuelle}

A partir des dialogues à bâtons rompus enregistrés récemment par les étudiants, sur cassette audio et sur cassette numérique vidéo, on a pu se rendre compte que la mimique-gestuelle est, comme l'intonation, partie intégrante de l'usage oral de la langue - et plus précisément d'une langue particulière - et qu'on ne saurait donc parler du geste en tant que phénomène «paraverbal» ou «co-verbal», ni non plus parler d'invariants qui transcenderaient la diversité des langues et des cultures. Les mimiquesgestuelles doivent être étudiées comme partie intégrante d'une langue dans son usage oral. Leur interprétation ne peut se faire, dans l'état actuel des recherches, que dans leur relation à une production langagière particulière. Pour parvenir à vérifier l'hypothèse qu'il pourrait exister des fonctions stables pour une association concomitante de paramètres des trois plans (morphosyntaxique, intonatif et mimicogestuel) dans une langue donnée, il est, bien entendu, nécessaire de procéder patiemment à des études de cas, pris isolément et confrontés les uns aux autres.

On a tout d'abord constaté que les mouvements de la tête, du regard et des mains ont une incidence plus large que les indices fournis par l'intonation et la syntaxe; ils ont une durée plus tenue. On a ensuite repéré que le début des mouvements de regard ou de mains se produit légèrement avant l'émission sonore que ces mouvements accompagnent. De là nous avons formulé l'hypothèse que ces mouvements de regard et des mains rendent visibles les opérations linguistiques en cours avant qu'elles ne se manifestent dans le discours. Ils se produisent effectivement en des moments-clés, repérables par rapport au début et à la fin des constituants du paragraphe, en parfaite complémentarité avec les deux autres plans. Ainsi le regard du parleur quitte régulièrement l'écouteur juste avant la production sonore du début du préambule, et revient vers lui avant la fin du rhème, souvent juste après le début du rhème.

(2) Corpus Québec: Deux étudiantes échangent leurs impressions de voyage à l'étranger. Après avoir évoqué son peu d'attrait pour les Etats-Unis, Marie parle de ce qu'elle a ressenti lors de son séjour au Québec.

Marie1- oh ça m'attire pas du tout ${ }^{\circ}$ les Etats-Unis ${ }^{\circ}$

Christelle1- ouais

Marie2- par contre le Québec c'est vraiment magnifique

Christelle2- ouais moi j'pense que ça m'plairait bien aussi

Tracé 2 : Marie3- $\{50\}$ et eub tu vois là j'ai <é> j'ai vraiment respiré $\{50\}$ quand j'y suis allée c'était euh $\{150\}$ le ca :::Ime pas d'pollution :: l'air pu :::r 
Un thème, deux thèmes, un préambule?

Tracé 2 et images : extrait du corpus Québec (Bouvet et Morel 2002, p.40-43)

ANAPROZ C: \CORPUS MAR. WAUU $8 \mathrm{~b} 22050 \mathrm{~Hz} 1 \mathrm{mn} 11 \mathrm{~s} .00$
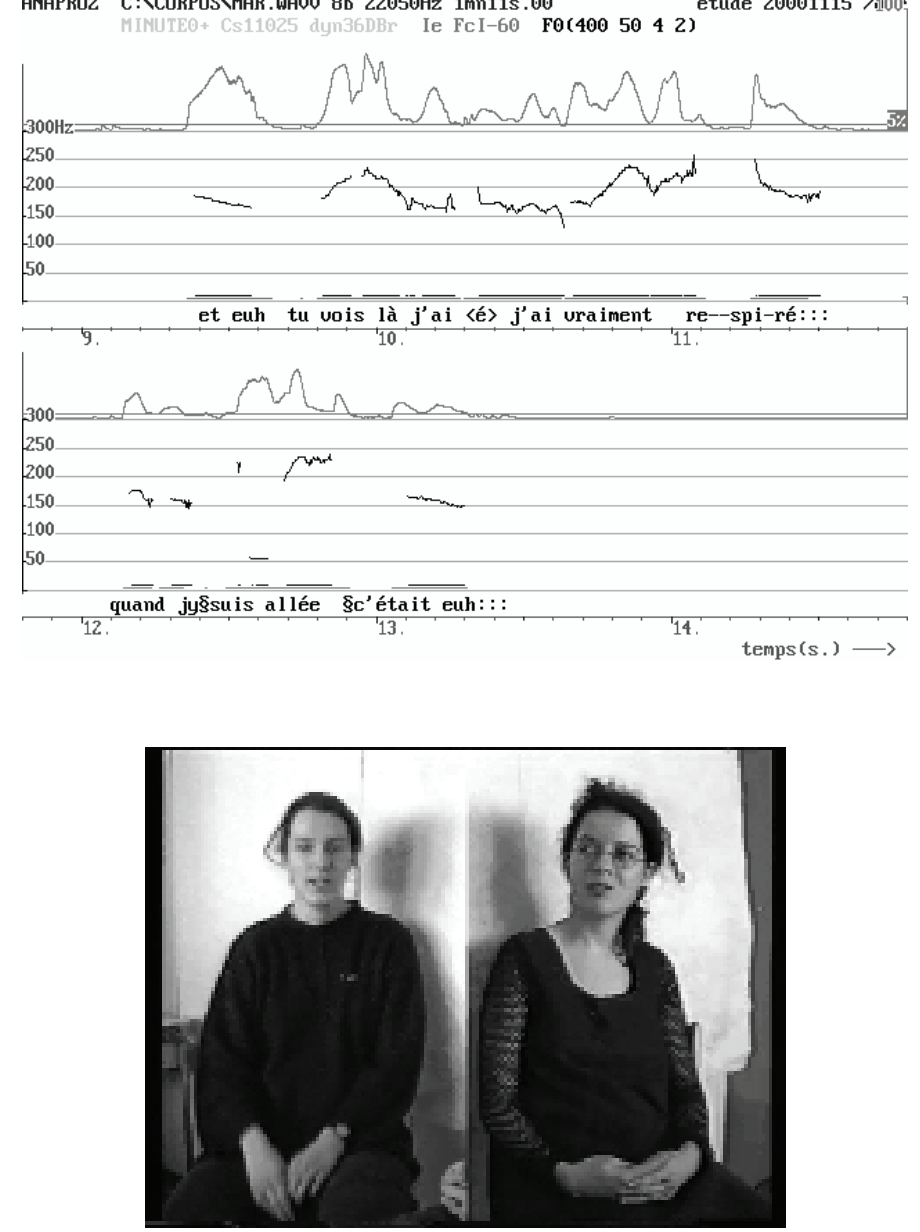

$<\mathrm{R}>$ et euh tu vois

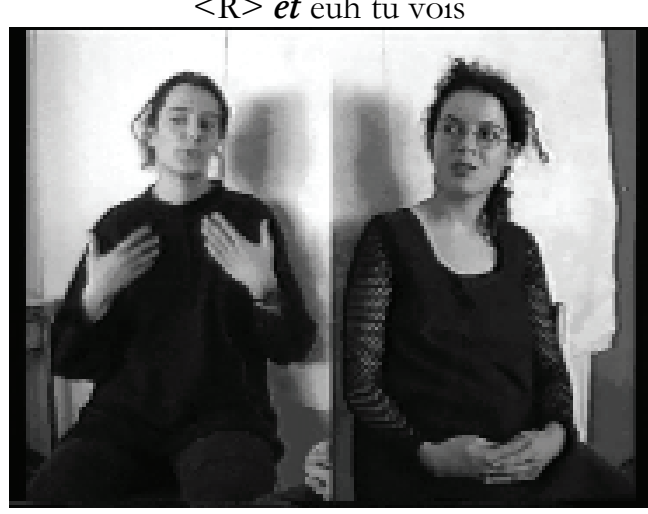

et euh $\boldsymbol{t} \boldsymbol{u}$ vois 
Mary-Annick Morel

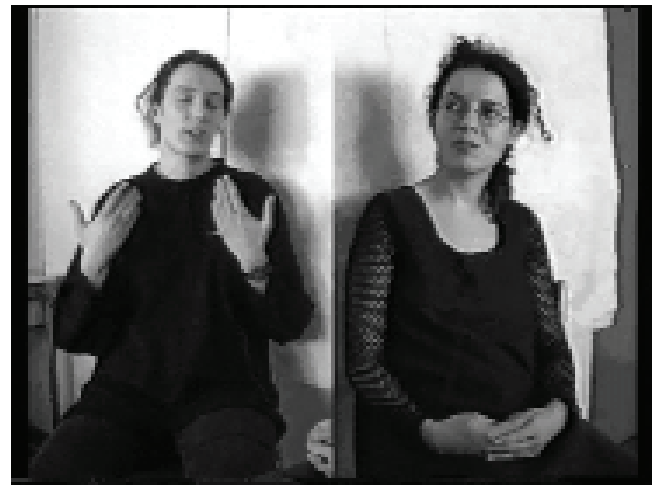

tu vois là $j$ 'ai

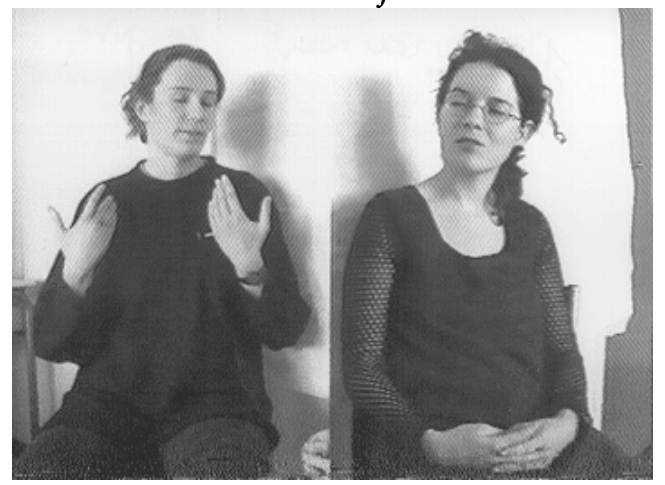

j’ai é j’ai vraiment respiré

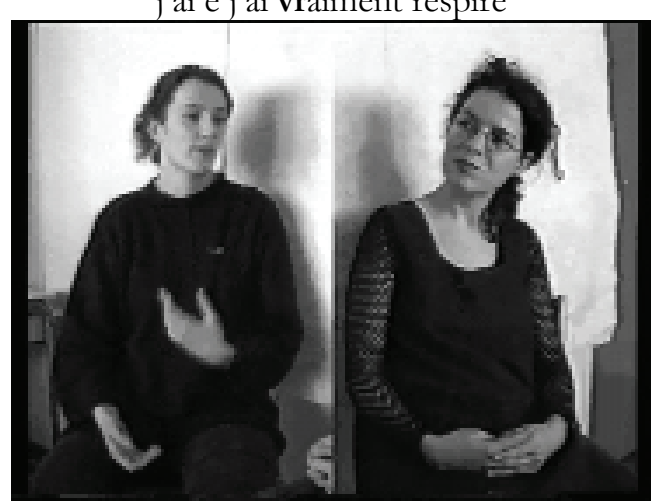

j’ai vraiment res-piré

Dans sa $3^{\mathrm{e}}$ intervention, Marie, après une pause silencieuse (normale) de $50 \mathrm{cs,}$ quitte du regard et de la tête Christelle, juste avant de prononcer le ligateur et eub (marque morphosyntaxique de thématisation de ce qui vient d'être dit) dont les propriétés intonatives sont: mélodie au niveau neutre (niveau moyen), intensité forte,

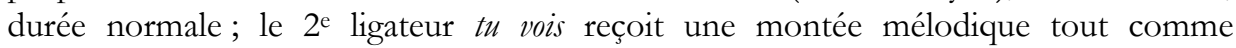
l'adverbe de cadrage spatio-temporel là (qui renvoie au domaine du Québec introduit dans le paragraphe précédent) : la montée mélodique marque ainsi l'anticipation d'une 
convergence des regards sur cet objet de discours. Puis vient le rhème différencié caractérisé par le redoublement de la marque de position égocentrée j'ai à un niveau mélodique bas, suivi d'un réhaussement en plage haute sur la $2^{\mathrm{e}}$ syllabe de vraiment, destiné à témoigner de la sincérité du jugement et de l'intensité du ressenti. A ce moment précis (syllabe «-ment») la tête revient en direction de Christelle, puis le regard revient au moment de la production de «res-» de respiré. La mélodie suit un mouvement bas-haut-moyen sur les 3 syllabes de respiré. Christelle de son côté fait de petites oscillations verticales de la tête, sans cesser de regarder Marie. Le $\mathrm{mm}$ qu'elle produit semble autorisé par la convergence des indices intonatifs (chute de l'intensité) et gestuels (retour de la tête et du regard) ; il vient se superposer à la suite des paroles de Marie quandj'y suis allée.

La répartition des marques des trois plans en des points différents du paragraphe intonatif assure une cohésion maximale aux segments successifs et témoigne d'une parfaite gestion de la coénonciation et de la colocution, chaque plan relayant l'autre dans le déroulement morphosyntaxique du discours et dans l'expression de la position coénonciative-colocutive.

\section{Principes de la théorie de la coénonciation / colocution / formulation}

L'analyse minutieuse de nombreux enregistrements transcrits a conduit à l'élaboration d'une théorie de la "coénonciation / colocution / formulation », qui permet de rendre compte des régularités rencontrées tant au plan morphosyntaxique qu'au plan intonatif et mimico-gestuel, et de retenir une unité de l'oral opératoire et aisément repérable, le paragraphe intonatif. A partir d'aménagements apportés par Laurent Danon-Boileau et moi-même à la théorie des opérations énonciatives d'Antoine Culioli, il a été possible de proposer des hypothèses sur la valeur iconique de base des indices intonatifs et sur la valeur conventionnelle qu'ils acquièrent lorsqu'on analyse leur fonctionnement conjoint. Des hypothèses comparables ont ensuite été développées pour rendre compte des fonctions de la mimique-gestuelle, dans les manifestations de la coénonciation / colocution / formulation.

\subsection{Les régularités intonatives $d u$ français}

Les variations des paramètres intonatifs (mélodie, intensité et durée) affectent régulièrement, en français, la syllabe finale des groupes. D'une manière générale, le français se caractérise par une tendance à l'isochronie des syllabes ${ }^{6}$ (durée des syllabes oscillant entre 12 et 20 centisecondes), des pauses silencieuses relativement courtes (40 à 60 centisecondes, environ une demi-seconde), une plage mélodique stable (F0 entre 70 et $250 \mathrm{Hertz}$ environ pour une voix masculine, et entre 150 et 400 Hertz pour une voix de femme) et une intensité plus forte en début de prise de parole.

\footnotetext{
${ }^{6}$ Cf. Paul Fraisse (1974) p. 149 «En français, le syllabisme est lié au principe de l'isosyllabisme, c'est-à-dire à l'égalité ou, plus exactement, à la non-différenciation des durées des syllabes. »
} 


\subsection{Les participants du dialogue : parleur et écouteur}

Nous avons choisi de désigner les participants d'un dialogue par les termes de « parleur» et d'« écouteur», qu'il faut entendre dans leur acception agentive de nom dérivé de verbe avec un suffixe marquant l'agent de l'action, à savoir

- parleur : « celui qui exerce l'activité de parler»

- écouteur : « celui qui exerce l'activité d'écouter».

Pour qu'un véritable dialogue s'instaure, il faut

(1) un minimum de bases communes et de consensus sur l'objet de discours et sur ses enjeux,

(2) un minimum de différenciation ou de singularisation sur le jugement que le parleur se prépare à porter sur cet objet de discours,

(3) un minimum de manifestations de l'activité d'écoute de la part de celui auquel les propos sont adressés.

\subsection{La coénonciation : énonciateur et coénonciateur}

Nous définissons la coénonciation comme l'anticipation par le parleur (alors envisagé comme énonciateur) des réactions possibles de l'écouteur, fondée sur le degré de connaissances partagées qu'il lui suppose. Le coénonciateur n'est pas la personne physique de l'écouteur. Le coénonciateur n'existe que dans l'esprit du parleurénonciateur. C'est l'auditoire que le parleur se forge pour les propos qu'il énonce.

Les variations dans la position coénonciative sont, dans notre théorie, explicitées par les variations de la mélodie, ainsi que par les changements d'orientation du regard. Il existe trois positions possibles pour le parleur-énonciateur :

- en côte à côte : l'énonciateur anticipe un consensus coénonciatif ou cherche à pallier un désaccord possible; la variation de la mélodie se fait vers le haut à la finale du constituant, tandis que le regard du parleur se détourne de l'écouteur avant le début de la production du préambule, pour revenir ensuite sur lui après le début du rhème,

- en face à face : l'énonciateur exprime une position qu'il juge différente de celle qu'il prête au coénonciateur: la variation de la mélodie se fait vers le bas et le regard du parleur reste rivé sur l'écouteur,

- en rupture : l'énonciateur adopte une position de repli sur soi ; il ne fait plus d'anticipation en direction du coénonciateur ; il y a absence de variations mélodiques ; la mélodie est basse et plate. Dans cette position égocentrée, le regard du parleur est détourné de l'écouteur, dirigé soit vers le bas (notamment lors d'une recherche liée à la formulation), soit vers le haut (lors d'une opération de conceptualisation et d'organisation de la structure discursive).

\subsection{La colocution : locuteur et colocuteur}

La colocution concerne plus précisément la gestion par le parleur (alors envisagé comme locuteur) du droit à la parole de chacun et les anticipations qu'il est amené à faire d'une éventuelle prise de parole de l'écouteur. Le colocuteur n'est pas (là non plus) la personne physique de l'écouteur. Le colocuteur n'existe que dans l'esprit du parleur-locuteur, en tant que rival possible dans l'exercice de la parole. 
Les variations dans la position colocutive sont, dans notre théorie toujours, explicitées par les variations de l'intensité (réhaussement ou chute) ou par l'absence de variation dans l'intensité. Il existe deux positions colocutives possibles pour le parleurlocuteur:

- s'il anticipe une prise de parole de l'écouteur et manifeste son désir de garder le droit à la parole en bloquant toute intervention possible de l'écouteur, il réhausse alors l'intensité et détourne la tête de l'écouteur,

- s'il prévoit une réaction sonore de l'écouteur (cf. réponse à une question ou simple marque d'écoute), ou s'il ne se soucie plus de préserver son droit à la parole, il laisse alors chuter l'intensité et tourne (ou maintient) sa tête en direction de l'écouteur. L'écouteur se sent alors autorisé à s'octroyer le droit à la parole.

\subsection{La formulation}

La gestion de la formulation par le parleur, face à un écouteur dont il anticipe les positions coénonciative et colocutive, est indissociable (en français du moins) de l'occupation maximale du canal sonore. L'allongement dans la durée de certaines syllabes est alors l'indice pour l'écouteur que le parleur aurait plus à dire sur l'objet de discours que ce qui vient effectivement d'être dit. En fonction des autres indices intonatifs, l'allongement est interprétable

- soit comme marque du travail de formulation et désir de continuation : la mélodie se situe à un niveau moyen et l'intensité est maintenue; l'allongement se produit sur des sons qui ne sont pas en eux-mêmes référentiels (finale de syntagme, mot-outil ou « euh »).

- soit comme une exclamation implicative destinée à qualifier une position supposée partagée par l'écouteur et à établir un lien avec l'explicitation qui va suivre ; l'allongement est accompagné d'une variation de la mélodie de type bas-haut-bas.

Le français dispose d'autres indices pour accompagner le travail de formulation, qui relèvent aussi du principe d'occupation du canal sonore : le « euh » dit d'hésitation, ou la réduplication des mots-outils.

Certains gestes réalisés avec les mains peuvent également être interprétés dans leur fonction d'aide à la gestion de la formulation. Ils permettent, en effet, d'opérer la localisation déictique d'un référent dans l'espace réel de l'échange, de scander la recherche de la formulation adéquate de ce que le parleur se prépare à dire et ainsi de gérer la poursuite du dialogue.

\subsection{La pause silencieuse}

Quant à la pause silencieuse, il a depuis longtemps été souligné qu'elle n'a pas de position fixe, qu'elle ne se présente pas nécessairement à la fin d'un constituant syntaxique, mais qu'elle est au contraire fondamentalement liée à la construction du sens.

La pause est nécessaire du côté du parleur ; elle sert à homogénéiser la séquence qu'elle vient clore et à lui conférer une unité sémantique. Mais elle est surtout et avant tout nécessaire du côté de l'écouteur. Ce dernier a en effet besoin régulièrement d'un temps de silence pour construire le sens de ce qu'il vient d'entendre. Un discours comportant peu de pauses ou des pauses trop brèves est un discours difficile à suivre. 
Mary-Annick Morel

\section{Le paragraphe intonatif, unité d'analyse du dialogue oral}

Le paragraphe intonatif s'identifie par la chute conjointe de la mélodie et de l'intensité sur la syllabe finale d'un groupe intonatif: cf. tracé 1 (11-12) sur «-llet» de billet. La prise en compte de la direction de la tête et du regard permet en outre, le plus souvent, de repérer la démarcation initiale du paragraphe : tête et regard du parleur se détournent de façon régulière de l'écouteur juste avant le début du préambule, c'est le cas dans l'exemple (2) tracé 2 (9-10) juste avant la production du et euh.

\subsection{Le paragraphe intonatif de type binaire}

Le paragraphe intonatif simple de type binaire comporte, de façon régulière, deux constituants: le préambule et le rhème.

Le paragraphe binaire peut se complexifier, grâce à la remontée de la mélodie à la finale d'un rhème, qui opère la recatégorisation de ce rhème en préambule pour la suite. Un paragraphe complexe présente donc plusieurs rhèmes successifs, voire plusieurs ensembles de <préambule + rhème $>$.

Nous avons également été amenés à définir une unité plus large que le paragraphe, que nous avons appelée «hyperparagraphe» (cf. Bouvet et Morel 2002), lorsque le regard du parleur se maintient sur l'écouteur au début du préambule suivant. Ceci se produit chaque fois que le parleur, anticipant une incompréhension de l'écouteur, juge bon d'introduire une glose sur son discours précédent. Le maintien du regard sur le préambule-cadre quand j'y suis allée jusqu'à la fin de la longue pause (une seconde et demie) s'explique, en effet, par le fait que la séquence s'inscrit dans le même cadre coénonciatif et thématique que ce qui précède et eub tu vois là.

(2) Corpus Québec : Tracé 2: Marie3- $\{50\}$ et eub tu vois là j’ai <é> j'ai vraiment respiré $\{50\}$ quand j’y suis allée c'était euh $\{150\}$ le ca::lme:::

\subsubsection{Le préambule}

Le préambule en français est souvent très décondensé, c'est là une des particularités du français, qui ne se retrouve pas dans un grand nombre d'autres langues (cf. Conway 2005). Il présente une succession de sous-constituants de fonctions différentes, donnés dans un ordre relativement fixe et dont l'ensemble est doté d'une forte montée mélodique sur la dernière syllabe. Le préambule du paragraphe ne se réduit donc pas à un seul constituant «thème » destiné à indiquer le référent sur lequel va porter le jugement énoncé dans le rhème qui suit. Les premiers éléments du préambule ont, en fait, pour fonction de mettre en place progressivement un consensus coénonciatif, un préalable commun nécessaire pour l'interprétation de ce qui va suivre. Ils ont pour rôle d'expliciter en premier l'articulation à ce qui vient d'être dit («ligateur» au sens de Bader 1986) ainsi que la position modale du parleur («modus» au sens de Bally 1932). Ils ont pour rôle aussi de simplifier le «travail d'écoute» de l'interlocuteur. C'est ce qui explique le dédoublement fréquent du constituant thématique en un élément de cadrage (" cadre» de type spatio-temporel ou délimitant un domaine interprétatif) suivi d'un "support lexical disjoint» énonçant l'élément référentiel destiné à servir de base au jugement énoncé dans le rhème, dont le 
statut d'argument sera assuré par la reprise anaphorique sous forme de pronom auprès $\mathrm{du}$ verbe $\mathrm{du}$ rhème. Un échange réussi repose toujours sur un préalable consensuellement partagé. C'est de l'anticipation des réactions possibles de l'écouteur que découle la succession des constituants discursifs du préambule, et notamment le dédoublement du constituant thématique.

Préambule-type du paragraphe intonatif

ligateur + point de vue + modus dissocié + cadre + support lexical disjoint (syntagme nominal ou 'il y a SN')

(3) Corpus Amour : Le dialogue met en jeu trois interlocuteurs, dont deux ont lu un livre sur les différences de conception de l'amour entre l'orient et l'occident. Le locuteur soutient la conception défendue par le livre, à savoir que l'amour transforme les personnes et rayonne autour des deux personnes qui s'aiment.

Pascal : tu vois moi je trouve fantastique que dans le livre le béros il reste pas comme ça il se transforme il se transforme complètement

Préambule ligateur : tu vois

point de vue : moi

modus dissocié : je trouve fantastique

cadre : que dans le livre

support lexical disjoint : le héros

Rhèmes 1-2-3 il reste pas comme ça - il se transforme- il se transforme complètement

(4) Corpus Vol de manteau : Deux étudiantes amies, Karine et Delphine, discutent à bâtons rompus. Par association d'idées Karine en vient à raconter une anecdote concernant les circonstances dans lesquelles une de leurs connaissances s'est fait voler son manteau.

Karine : non mais y a comme ça là:: e.:: t(out) à l'beure hein quand on vu la:.:.:. la scur de Laurent $\{35\}$ e(lle) s'est fait piquer son manteau $\{60\}$ e $\{20\}$ chez: e:::: $\{20\}$ chez Kookaï ou j' sais plus quoi $\{20\}$ \Delphine- ah ouais $\$ pendant qu'elle essayait un truc $\{150\}$ §Delphine 43 - ben c'est pas d' bol ${ }^{\circ} \mathrm{ça}^{\circ} \int[\ldots]$

Delphine 44 : e(lle)s étaient dans les cabines non ou sur e dans les rayons?

Karine 53 : non non e(lle)s étaient dans les rayons $\{30\}$ pis tu sais e::: è-e(lles) regardai(en)t une au/ $<$.> un autre manteau $\{20\}$ donc elle enlève le sien et e(lle) le pose: e:: bon ben sur le:

Delphine 45 : onais sur le cintre à côté e ouais

Karine 54 : (b) tu sais sur le cin:tre tu fais bon vraiment tu fais pas attention $\{70\}$

$\$ 1$ Préambule1 ligateur: non mais

modus : y a comme ça

cadre1 : là:: e::::

cadre2+ponctuant : t(out) à l'beure bein

point de vue+support lexical disjoint : quand on vu la::.:.: la scur de Laurent $\{35\}$ 
Mary-Annick Morel

Rbème 1 e(lle) s'est fait piquer son manteau $\{60\}$ e $\{20\}$ chez: e:::: $\{20\}$ chez Kookaï ou j' sais plus quoi $\{20\}$

Réaction sonore de l'écouteuse ab onais

Rhème 2 pendant qu'elle essayait un truc $\{150\}$

Préambule - rbème - postrbème ben + c'est pas d' bol $+{ }^{\circ} \mathrm{ça}^{\circ}$

Tracé 3 : début de la narration Vol de manteau ex. (4)
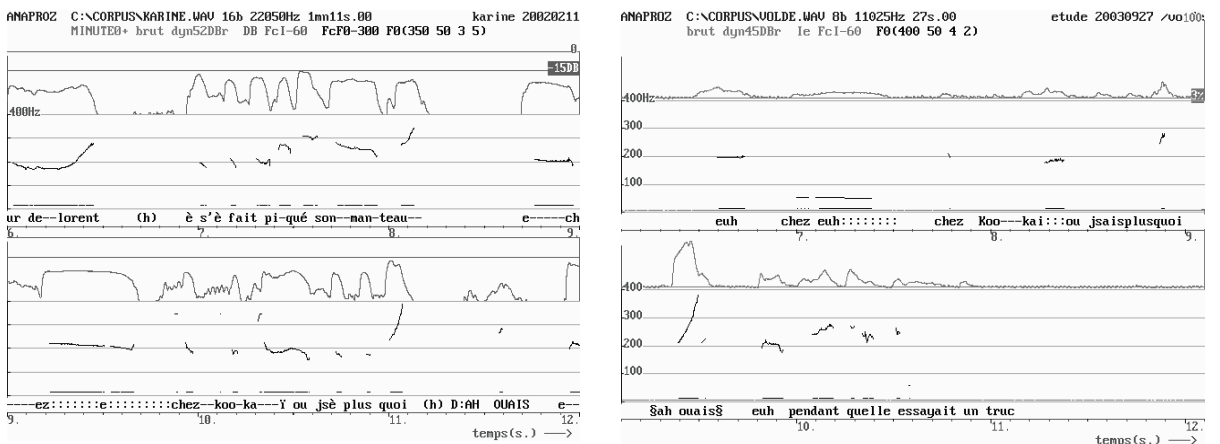

(5) Corpus Alain Rey : Il s'agit d'un débat télévisé autour de la langue française. L'animateur de l'émission, Frédéric Ferney, après avoir fait un tour de table pour recueillir les opinions des participants concernant la langue française, se prépare à donner la parole à Alain Rey, à propos de la nouvelle édition du Grand Robert.

Frédéric Ferney1: vous Alain Rey ob j'avoue qu'c'est vous le plus optimiste puisque vous vous dites en gros si le français est en crise en danger $\{30\}$ c'est la preuve que c'est une langue vi vante $\{40\}$

Préambule1 support lexical disjoint-apostrophe : vous Alain Rey + ligateur : ob

$$
\text { modus : j'avoue qu' }
$$

Rhème1 recatégorisé en préambule : c'est vous + le plus optimiste

Préambule2 ligateur : puisque

point de vue1 : vous

modus-point de vue 2 : vous dites en gros

cadre-support lexical disjoint : si le français est en crise en danser $\{30\}$

Rhème 2 c'est la preuve que c'est une langue vi ${ }_{\text {vante }}$

En premier lieu viennent les éléments qui explicitent la position coénonciative et l'attitude modale du parleur et qui correspondent à trois types de sous-constituants distincts : ligateur + point de vue + modus dissocié.

Le français présente une très grande variété de formes de ligateurs, dont la fonction oscille entre la spécification des anticipations coénonciatives du parleur (tu vois, non, non non, mais, non mais, tu sais, ouais, ab ouais, ob, puisque, écoute, vous savez, en tout 
cas, de toutes façons, en fait, par contre...) et/ou le marquage de la cohésion et de la progression textuelle (ben, bon, donc, pis, alors, et $p(u) i$ s, d'abord, ensuite ...).

Immédiatement après le ligateur viennent les marques de point de vue qui servent à souligner l'identité de l'énonciateur qui sert de caution à ce qui va être dit (moi, quand on a vu, vous, vous dites en gros, pour eux, elle a dit ...) ; elles sont parfois associées à la caractérisation de la valeur de la modalité et sont alors repérables dans l'emploi de certains pronoms du constituant suivant, le modus dissocié (je trouve, c'est vrai ..., ) où se trouve explicitée la position modale (épistémique : je trouve, vraiment, javone, y a comme ça ou appréciative : fantastique) du parleur. Les modulations dans le point de vue et dans l'attitude modale sont extrêmement fréquentes dans le déroulement du dialogue en français.

Un deuxième ensemble de sous-constituants du préambule présente les constructions référentielles qui vont servir de fondement "thématique » à la prédication exprimée dans le rhème. Il s'agit alors de construire en premier lieu le cadrage thématique (repérage spatio-temporel, délimitation d'une zone de sens, d'un domaine référentiel restreignant l'espace interprétatif de la suite : dans le livre, là, tout à l'heure, si le français est en crise en danger), puis en deuxième lieu d'énoncer ce qui va précisément servir de base(de "thème ») au rhème qui suit. Le dernier sous-constituant du préambule, que nous appelons «support lexical disjoint» peut présenter deux formes syntaxiques : soit il est constitué d'un syntagme nominal qui est repris par un pronom personnel ou démonstratif dans le rhème (cf. ex. (5) rhème $2 c^{\prime}$ ), soit il est introduit par un présentatif existentiel ou un verbe de perception (ily $a, j$ 'ai..., quand on a vu la scur de Laurent) dont le relais anaphorique est assuré par un pronom relatif (ou par un pronom personnel, cf. ex. (6) rhème1 elle).

(6) Corpus Mythomanes : La conversation tourne autour de la mythomanie. L'une des deux interlocutrices se souvient soudain qu'elle a eu une expérience indirecte d'une personne mythomane. mythomane

Monique : ben oui $\{25\}$ j'avais une:: $\{20\}$ une ancienne collègue d/ la môme elle était

préambule 1 ligateur : ben oni

cadre : j'avais une:: $\{20\}$ une ancienne collègue

support lexical disjoint : $d /$ la môme

rbème 1 elle était mythomane

Le préambule présente rarement l'ensemble des constituants analysés ci-dessus, mais il se caractérise par un très large éventail de formes possibles. Le préambule est, en fait, destiné à simplifier le travail d'écoute de l'interlocuteur, et à assurer au maximum le bon fonctionnement de la coénonciation. Un dialogue où les préambules de l'un des interlocuteurs sont trop réduits présente un risque de dysfonctionnement.

\subsubsection{Le rbème}

Le deuxième constituant du paragraphe, que nous appelons " rhème », est en règle générale assez bref en français. Ceci se comprend aisément, étant donné le travail de décondensation des opérations coénonciatives, modales et référentielles opéré dans 
le préambule. Il est construit autour d'un verbe conjugué doté d'un sujet pronominal, ou à partir d'un présentatif (c'est ou il y a) ; il peut aussi se présenter sans verbe (SN, adjectif ou adverbe seul). Le rhème est parfois terminé par un ponctuant (quoi, hein, ouais, voila), qui réitère en l'explicitant la position coénonciative affichée tout au long du paragraphe. Le rhème n'a pas pour fonction, comme on le dit souvent, de présenter le contenu le plus informatif (c'est plutôt au préambule qu'est assigné ce rôle). Il a plus exactement pour rôle de préciser la position différenciée du parleur par rapport à la pensée qu'il prête à l'écouteur-coénonciateur à ce moment précis de l'échange.

Le rhème ne présente qu'une relation syntaxique à la fois. Ainsi dans l'exemple (3) le parleur produit trois rhèmes successifs qui introduisent chacun un élément supplémentaire à la relation syntaxique initiale : il reste pas comme ça / il se transforme / il se transforme complètement, et dans l'exemple (4) Tracé 3 le circonstant temporel pendant qu'elle essayait un truc prend la valeur d'un rhème autonome du fait qu'il est intonativement autonomisé par rapport au rhème précédent par la remontée mélodique sur quoi, qui recatégorise le rhème en préambule pour la suite, ce qui est entériné par la manifestation sonore de l'écouteuse ah ouais cf. Tracé 3.

Le rhème se caractérise en outre par le retour de la tête et du regard du parleur en direction de l'écouteur. Il est de fait indispensable pour le parleur de constater que l'écouteur maintient son regard pour assurer la cohésion de ce qu'il dit, et aussi de voir si ses anticipations coénonciatives se vérifient dans l'attitude générale (hochements de tête...) et la mimique de l'écouteur (sourire, direction du regard...).

\subsection{Le paragraphe de type ternaire}

Cette deuxième variété de paragraphe intonatif présente une structure fixe, à trois constituants <préambule + rhème + postrhème $>$ (parfois réduits à deux $\left(<\right.$ rhème + postrhème $>\mathrm{cf}$. Ils sont fous ${ }^{\circ}$ ces Romains $\left.{ }^{\circ}\right)$. Il se caractérise par la brièveté de son préambule cf. ob dans (2) Marie 1 oh ça m'attire pas du tout ${ }^{\circ}$ les Etats-Unis ${ }^{\circ}$ et ben dans (4) ben c'est pas d'bol ${ }^{\circ} \mathrm{g}^{\circ}$. Le paragraphe ternaire est très typé sur le plan intonatif. La syllabe finale du rhème est dotée d'une forte hauteur mélodique (sur bol de pas d'boh), alors que le postrhème est donné en mélodie plus basse, et ne remonte pas sur la syllabe finale. Le postrhème se caractérise par un faisceau de propriétés indissociables : il n'est jamais séparé du rhème par une pause, il comporte au maximum sept syllabes, il est de nature nominale (il est anticipé par un substitut cataphorique dans le rhème), il ne présente jamais de verbe conjugué. Il marque un positionnement égocentré du parleur à propos d'un référent construit dans l'échange préalable (cf. (2) les Etats-Unis et (4) $\varsigma$ a) dont il pense qu'il est le seul à pouvoir énoncer une propriété qui échappe à la connaissance de l'écouteur. Il introduit, de ce fait, le plus souvent, une rupture dans le continu du dialogue (clôture d'un épisode narratif dans (2) et (4)). 
Tracé 4 : Corpus Vol de manteau

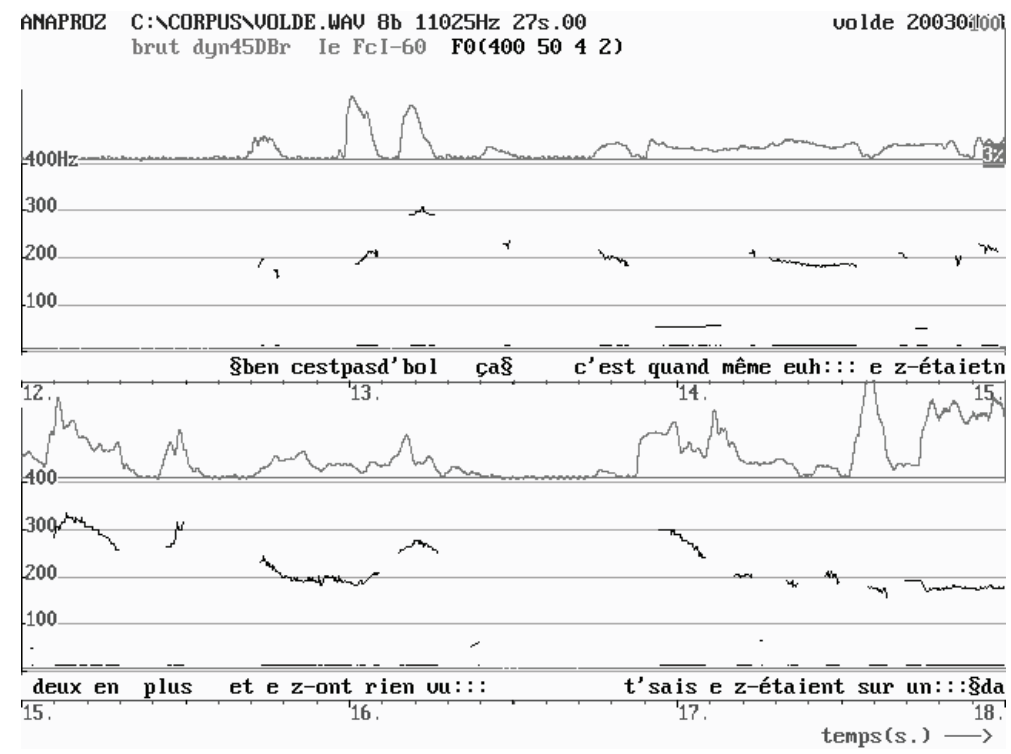

\section{Les productions sonores de l'écouteur}

Dans un dialogue en français, l'écouteur sait qu'il se doit de se manifester de façon sonore. Il jalonne donc son écoute de productions sonores plus ou moins étoffées, qui font partie de l'organisation interne du dialogue ${ }^{7}$, allant du simple mm / onais et des interjections primaires ah / oh au jugement de valeur ouais moi jppense que ça m’plairait bien aussi (cf. (2) Corpus Québec, Christelle 2) en passant par des interjections plus élaborées ah bon, des syntagmes qualifiants, voire des exclamations ben c'est pas d'bol ça (cf. (4) Corpus Vol de manteau, Delphine 43, tracé 4) ou des questions. Il ne se manifeste toutefois pas à n'importe quel moment. L'observation des conditions d'apparition des manifestations sonores de l'écouteur a permis de dégager un certain nombre de régularités.

- Première régularité: pour que l'écouteur se manifeste d'une manière quelconque, il faut qu'il y ait eu une chute d'intensité dans le discours du parleur.

- Deuxième régularité : délai de 40 cs. Entre la fin d'un groupe sémantiquement homogène dans le discours du parleur et une production sonore minimale ( $m m$, bien sûr etc.) de la part de l'écouteur, il faut que se soit écoulé un laps de temps de 40cs (cf. Tracé 5 bis et 5 ter Corpus CRS ci-dessous - Tatiana (2-3) et (8-9) produit un $m m 40$ cs après histoire qui termine le cadre 1 du préambule du récit et après l'métro qui clot le support lexical disjoint). Cette durée correspond au temps de la pause en français. Elle

\footnotetext{
${ }^{7}$ Le balisage du dialogue par des productions sonores de l'écouteur s'observe aussi dans d'autres langues. Les «mm » et les « yeah » sont, entre autres, fréquents en anglais, cf. Gardner R., 2001.
} 
Mary-Annick Morel

est aussi celle que les psychologues donnent pour la mémoire à court terme. Dans notre théorie, elle correspond au temps nécessaire à l'écouteur pour construire le sens de ce qu'il vient d'entendre.

- Troisième régularité : délai de $70 \mathrm{cs}$. Ce temps de réaction passe à $70 \mathrm{cs}$ ou plus (entre une seconde et une seconde et demie - 100 cs et $150 \mathrm{cs}$ ) lorsque l'écouteur produit une manifestation plus étoffée. Au delà des $40 \mathrm{cs}$ requis pour la simple compréhension des propos, le délai supplémentaire correspond au temps permettant d'organiser la formulation d'un jugement (cf. Tracés 3 et 4 ci-dessus - Tatiana produit son jugement réactif ben c'est pas d'bol ça 150 cs après la fin du rhème 2 pendant qu'elle essayait un truc). On peut ainsi comprendre pourquoi les manifestations sonores de l'écouteur ne se situent pas toujours au moment d'une pause silencieuse du parleur. Si ce dernier poursuit sans pause son discours après une chute d'intensité, cela provoque obligatoirement une superposition de la parole de l'écouteur à la sienne (cf. tracé 5 bis, le $m m$ de Tatiana (2-3) se superpose à la glose de Jane le tru::c).

- Quatrième régularité: le regard et la tête du parleur doivent être dirigés vers l'écouteur.

Le tracé 5bis de l'exemple (7) montre très clairement comment l'écouteuse, Tatiana, ponctue très régulièrement la mise en place par la narratrice, Jane, des données du préambule de son récit, par des $m m$ très sonorisés. La mélodie montante des $\mathrm{mm}$ accompagne son écoute consensuelle et manifeste le bien-fondé des anticipations coénonciatives consensuelles de Jane, à la suite des sous-constituants principaux du préambule : du groupe ligateur + point de vue + premier cadre ab j'ai appris une histoire, puis du cadre 2 à propos des CRS, et enfin du support lexical disjoint une nana qui est lui-même cadré par le premier des deux rhèmes coordonnés qui suivent donc c'est une nana elle prend l'métro.

\section{(7) Corpus CRS (début)}

Jane- ah j'ai appris une histoi::re le tru:: $\int \mathbf{m} \boldsymbol{m} \int c\{50\}$ à propos des cé-èr-e:ss:: $\{30\}$ $\lceil\mathbf{m m} \$ c'est une fille qui m'racontait ça::: $\{60\}$ donc c'est une nana: elle prend l'métro:: $\{40\}$ \mm $\int$ et elle a pas son billet donc elle est en fraude vis-à-vis de la légis $\leqq \underline{\mathbf{m m}} \leqq$ lation de la $\mathrm{RATP}^{\circ}$ enfin le truc logique quoi ${ }^{\circ}$

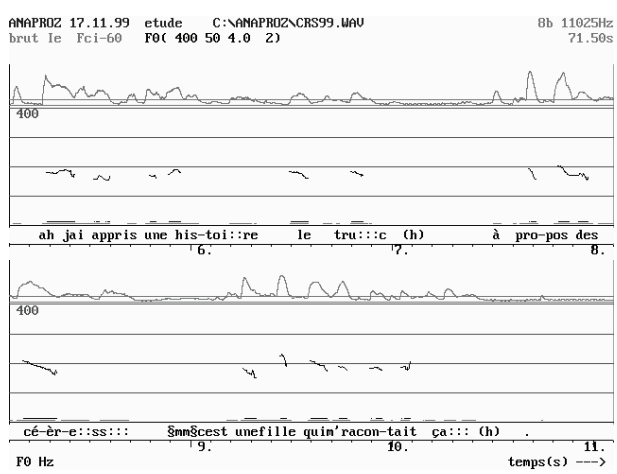

Tracé 5 : Corpus CRS - parleuse : Jane

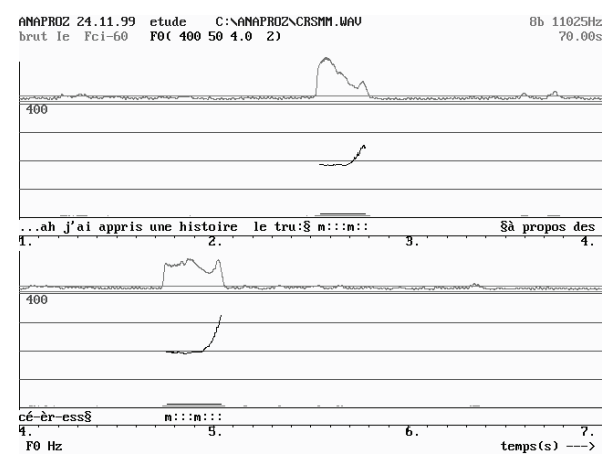

Tracé 5bis : Corpus CRS - écouteuse

Tatiana $\mathrm{mm}$ 
Tracé 5ter : Corpus CRS - écouteuse : Tatiana $\mathrm{mm}$

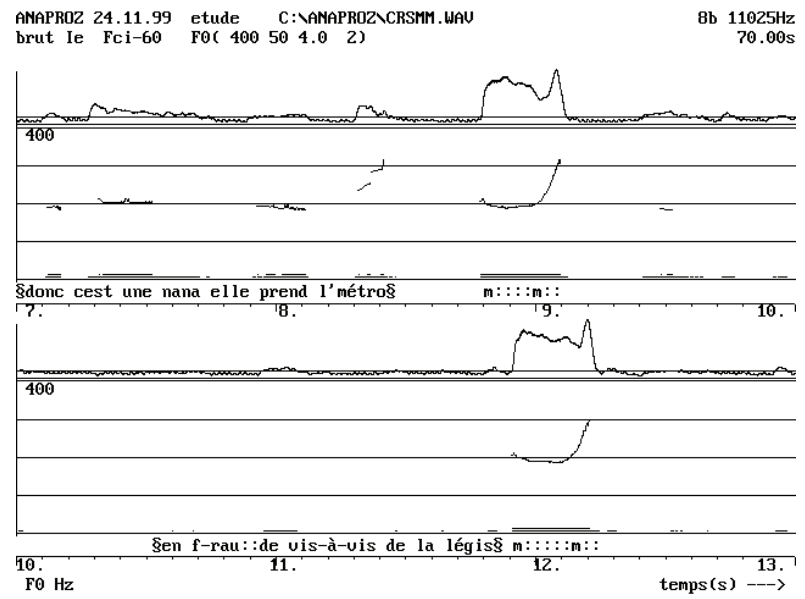

\section{Conclusion}

La prise en compte de la dimension multimodale de la parole et l'analyse conjointe des indices morphosyntaxiques, intonatifs et mimico-gestuels, tant du côté du parleur que du côté de l'écouteur, ont permis de circonscrire un certain nombre de propriétés du dialogue en français et de ses règles de bon fonctionnement. Il n'a pas été possible de les exposer toutes dans cette brève présentation. Je me contenterai de récapituler les points les plus caractéristiques du français.

L'unité de structuration orale, le paragraphe intonatif, se démarque à la finale par la chute conjointe de la mélodie et de l'intensité, mais il se démarque aussi à l'initiale par le départ du regard du parleur qui quitte l'écouteur avant la production du ligateur à l'initiale du paragraphe. Le paragraphe intonatif présente en général deux constituants discursifs : le préambule et le rhème. Le préambule est le plus souvent décondensé. Il sert en premier lieu à expliciter l'attitude coénonciative et la position modale du parleur, puis à délimiter progressivement le domaine référentiel thématique (cadre et support lexical disjoint) sur lequel le rhème va s'appuyer. Le rhème est souvent bref, il ne présente qu'une relation syntaxique à la fois, il sert à exprimer la position différenciée du parleur sur l'objet de discours à ce moment de l'échange. La complexification du paragraphe oral se fait par la remontée de la mélodie à la finale du rhème qui recatégorise celui-ci en préambule pour la suite.

La coénonciation est conçue comme l'anticipation faite par le parleur des attentes de l'écouteur, de son degré de connaissance, de ses réactions potentielles. Les modulations dans la coénonciation sont marquées de façon iconique par les mouvements de la mélodie : la montée mélodique traduit un mouvement vers l'autre, alors que la descente accompagne le repli sur soi.

Dans un dialogue en français il n'y a pas de place pour un silence prolongé. La pause silencieuse est relativement brève (une demi-seconde); elle est indispensable 
pour permettre à l'écouteur de construire le sens de la séquence qu'elle vient clore. Pour gérer une difficulté momentanée dans la formulation, le parleur se doit d'occuper au maximum le canal sonore, il dispose pour ce faire du euh, de la réduplication du mot-outil et de l'allongement de ce dernier.

L'attitude coénonciative du parleur se manifeste aussi par les mouvements dans la direction de son regard. Il quitte régulièrement des yeux celui auquel il adresse son discours, le temps de la production vocale des données informatives, et notamment des deux sous-constituants du cadrage thématique, qui permettront de construire entre eux une base d'attention partagée. L'absence de regard du parleur sur l'écouteur vient ainsi renforcer l'homogénéisation des deux constituants thématiques du préambule, le «cadre » et le «support lexical disjoint », déjà assurée par la remontée mélodique à la finale de chacun d'eux. Le retour du regard du parleur avant la fin du rhème (au début ou au milieu) lui permet de prendre connaissance des réactions de l'écouteur (consensus, désaccord ou incompréhension). Lorsque, au contraire, il se trouve face à une difficulté de formulation, son regard se détourne systématiquement de l'écouteur.

Quant à celui auquel le discours est adressé, il se doit de manifester sa bonne écoute de façon sonore et mimico-gestuelle. Il maintient son regard en direction du parleur, lorsque ce dernier parle. Recevoir la parole, ce n'est pas seulement écouter les émissions sonores de celui qui parle, c'est aussi regarder son activité corporelle lors de la production de son discours. L'enveloppe mélodique des réactions sonores de l'écouteur permet également au parleur de moduler ses anticipations coénonciatives. 


\section{BIBLIOGRAPHIE}

BADER, F., 1986, «Structure de l'énoncé indo-européen », Bulletin de la Société de Linguistique de Paris, Tome LXXXI, Peeters, Louvain, p. 71-120.

BALly, Ch., 1932, Linguistique générale et linguistique française, Leroux, Paris.

BLANCHE-BENVENISTE, Cl., et alii, 1990, Le français parlé. Etudes grammaticales, Editions du CNRS, Paris.

BOuvet, D., 2001, La dimension corporelle de la parole: les marques posturo-mimo-gestuelles de la parole, leurs aspects métonymiques et métaphoriques, et leur rôle au cours d'un récit, Peeters, Louvain, Collection de la Société de Linguistique de Paris.

Bouvet, D., \& MOREL, M.-A., 2002, Le ballet et la musique de la parole. Le geste et l'intonation dans le dialogue oral en français, Ophrys, Paris-Gap, Bibliothèque de Faits de Langues.

CANDEA, M., 2001, Contribution à l'étude des pauses silencieuses et phénomènes dits "d'bésitation 》en français oral spontané. Etude sur un corpus de récits en classe de français, Thèse de doctorat de Paris III (non publiée).

CONWAY, A., 2005, Le paragraphe oral en français L1, en suédois et en français L2. Etude syntaxique, prosodique et discursive, Etudes romanes de Lund 73, Lunds Universitet, Romanska Institutionen.

Danon-Boileau, L., \& MOrel, M.-A., 1994, «L'oral ou comment simplifier le travail d'écoute de l'autre », Cahiers du français contemporain $\mathrm{n}^{\circ} 1$, Simple - Simplification, F. Lapeyre (coord.), Paris, Didier-Erudition, CREDIF.

Danon-Boileau, L., \& Morel, M.-A., 2003, «L'écouteur vicariant », in Le sujet, J.-M. Merle coord., Ophrys, Bibliothèque de Faits de Langues, Paris-Gap, p.235-246.

FrAISSE, P., 1974, La psychologie du rythme, Paris, PUF.

GARDNER, R., 2001, When listeners talk. Response tokens and listener stance, John Benjamins Publ. Comp., Amsterdam / Philadelphia.

HASCOËT, N., 2005, Le geste et l'intonation à l'oral spontané : une étude de cas, Doctorat de l'université de Paris 5, dir. L. Danon-Boileau.

HiRST, D., \& Di CRISTO, A., (eds), 1998, Intonation Systems : A Survey of Twenty Languages, Cambridge Univ. Press.

La notion de paragraphe, 1985, Editions du CNRS, Paris, p. 23-40 et 41-51.

Morel, M.-A., \& Danon-Boileau, L., 1998, Grammaire de l'intonation. L'exemple du français oral, Ophrys, Paris-Gap, Bibliothèque de Faits de Langues.

Morel, M.-A., 2003, «Phrase ? Enoncé ? Paragraphe? Hyperparagraphe ? Quelles unités intonatives et discursives pour le dialogue oral en français ?", L'information Grammaticale $\mathrm{n}^{\circ} 98$, Peeters, Louvain, p. 39-47.

Morel, M.-A., 2003, «Valeur énonciative des changements de registre mélodique dans le dialogue oral en français », in Registre et voix sociale, Monique Demers éd., Editions Nota Bene, Québec, p. 125-157. 
Mary-Annick Morel

Orage’98, S.SAnTi, I.GuAitella, C.CAVE Et G.KonOpCZYNSKi (eds), 1998, Oralité et gestualité. Communication multimodale, interaction, Actes du colloque d'Aix, L'Harmattan, Paris.

OraGE’01, S.SAnTi, I.GuAitella, C.CAVE (eds), 2001, Oralité et gestualité. Interactions et comportements multimodaux dans la communication, Actes du colloque d'Aix, L'Harmatta, Paris. 